\title{
Histological Features of Intracranial Germinomas Not Disappearing Immediately After Radiotherapy
}

\author{
Satoshi UTSUKI, Hidehiro OKA, Yoshinori TANIZAKI, Koji KONDO, \\ Nobuyuki KAWANO*, and Kiyotaka FUJII \\ Department of Neurosurgery, Kitasato University School of Medicine, Sagamihara, \\ Kanagawa; *Isobe Clinic, Sagamihara, Kanagawa
}

\begin{abstract}
The histological features of germinomas were investigated to differentiate tumors which completely disappear immediately after irradiation and those that persist. Eighteen previously untreated patients underwent germinoma biopsy and irradiation or combined irradiation and chemotherapy. Four tumors were located only in the pineal gland, eight in the suprasellar region, two in multiple locations, one in the basal ganglia, and three in other regions. Histologically, the germinomas could be divided into type A found in 13 cases which consisted mainly of large neoplastic cells and small lymphocytes, the so-called two-cell pattern, and type $B$ found in five cases which consisted predominantly of fibrous tissue and granulomatous reaction containing occasional neoplastic cells. Follow-up computed tomography or magnetic resonance imaging showed the enhanced mass lesion disappeared in all cases of type A germinomas within 1 month after treatment, but persisted in all cases of type $B$ germinomas for at least 1 month. Type B tumors required up to 12 months to show complete radiographic resolution. Persistent germinomas consisted predominantly of fibrous tissue and granulomatous reaction containing occasional neoplastic cells.
\end{abstract}

Key words: fibrous tissue, germinoma, granulomatous reaction, magnetic resonance imaging, histology

\section{Introduction}

Radiation treatment of germinomas is very effective, with a dose as small as $20 \mathrm{~Gy}$ being curative., ${ }^{2,4,14)}$ Occasionally, the lesion does not completely disappear immediately after radiation therapy, even if the histological diagnosis is pure germinoma. ${ }^{11)}$ Other germ cell tumors are not radiosensitive and do not completely disappear after treatment with $20 \mathrm{~Gy}$ of radiation. ${ }^{3)}$ Therefore, if the histological diagnosis was pure germinoma, tumor which persists after radiation therapy may be pure germinoma that does not disappear immediately or germinoma containing non-germinomatous germ cell tumor. This difference has serious implications for the therapeutic strategy.

The present study investigated the histological characteristics of intracranial germinomas which did not resolve immediately after irradiation but required no further treatment.

Received July 1, 2005; Accepted April 16, 2006

\section{Material and Methods}

Eighteen patients with intracranial germinoma, 12 men and six women aged 6 to 44 years (mean 18 years), were selected from 57 cases of intracranial germ cell tumors, including cases with increased serum and/or cerebrospinal fluid (CSF) beta-human chorionic gonadotropin (HCG) levels, treated at the Department of Neurosurgery of Kitasato University Hospital between 1975 and 2002. Four tumors were located only in the pineal gland, eight in the suprasellar region, two in multiple locations, one in the basal ganglia, and three in other regions. Postoperative computed tomography (CT) scans or magnetic resonance (MR) images were available for all cases.

All specimens were fixed with $10 \%$ formalin and embedded in paraffin for hematoxylin and eosin staining, Masson trichrome staining, and immunostaining for placental alkaline phosphatase (PLAP), HCG, alpha-fetoprotein (AFP), leukocyte common antigen (LCA), CD20 (a B-cell marker), CD45 (a T-cell marker), and CD68 (a macrophage 


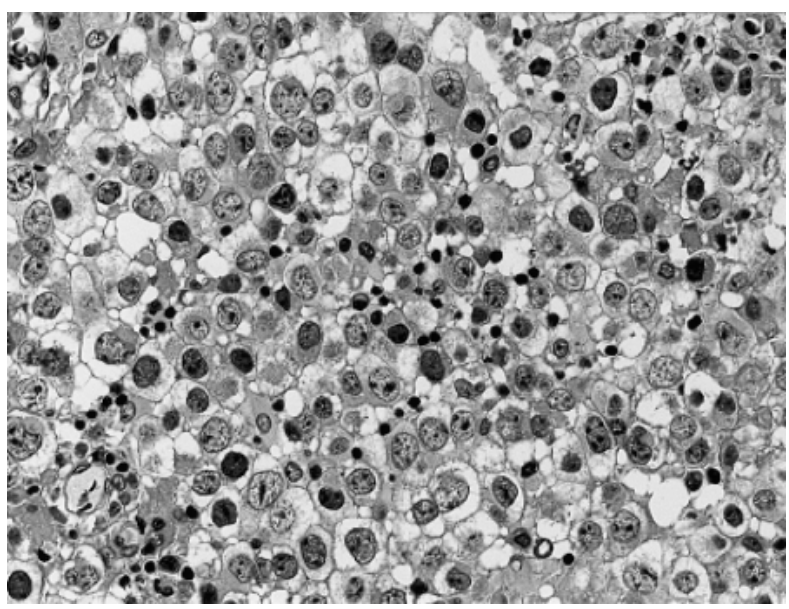

Fig. 1 Photomicrograph of a typical case of type A germinoma showing the so-called "two-cell pattern" of large neoplastic cells and infiltrated lymphocytes. Hematoxylin and eosin stain, $\times 400$.

marker). The serum titers of beta-HCG and AFP were examined in 12 patients. The CSF titers of betaHCG and AFP were examined in nine patients.

None of the patients were treated before biopsy. All patients until 1996 were treated with irradiation using local doses of 20-30 Gy for a total of 50-60 Gy of fractionated linear irradiation. Patients after 1996 were treated with irradiation using extended local doses of 20-30 Gy and two courses of chemotherapy (cisplatin and etoposide). The persistence of enhanced mass lesions was determined by CT or MR imaging performed within 1 month after treatment ended and repeated until complete resolution of the mass occurred.

\section{Results}

None of the specimens were immunoreactive for HCG and AFP and no syncytiotrophoblastic giant cells were observed. Almost all tumor cells were immunoreactive for PLAP and small cells were immunoreactive for LCA and CD45. A few cells were immunoreactive for CD68 except for remarkably granulomatous reactive specimens. All histological diagnoses were pure germinoma.

The germinomas could be divided into two histological types. The most common type A $(13$ cases) consisted mainly of sheets or 'nests' of large neoplastic germ cells and small lymphocytes, with a typical “two-cell pattern" (Fig. 1). Type B (5 cases) consisted predominantly of granulomatous inflammation and fibrous tissue (Fig. 2A) with occasional or rare neoplastic germ cells immunoreactive for

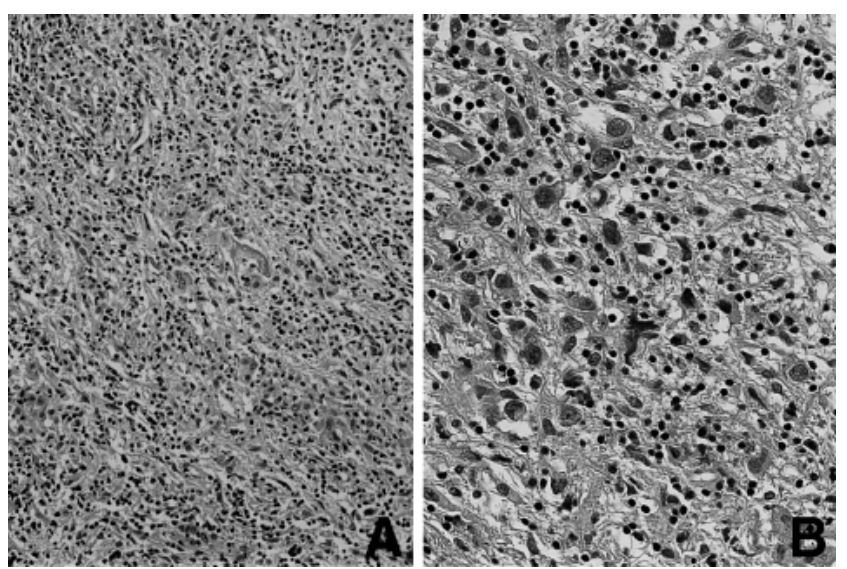

Fig. 2 Photomicrographs of a typical case of type $B$ germinoma showing epithelioid histiocytes and lymphocyte invasion with a fibrous background (A), and a few large cytoplasmrich neoplastic cells with lymphoid infiltrates (B). Hematoxylin and eosin stain, A: $\times 100, B: \times 400$.

Table 1 Summary of patients with types A and B germinomas

\begin{tabular}{lcc}
\hline & Type A (n= 13) & Type B (n = 5) \\
\hline Age (years) & $6-44$ (mean 18) & $8-28$ (mean 19) \\
Sex (male:female) & $9: 4$ & $3: 2$ \\
Primary location & & \\
$\quad$ pineal (n = 4) & 4 & 0 \\
suprasellar (n $=8)$ & 6 & 2 \\
multiple (n = 2) & 1 & 1 \\
basal ganglia (n $=1)$ & 1 & 0 \\
others (n = 3) & 1 & 2 \\
\hline
\end{tabular}

PLAP (Fig. 2B). Surgical specimens consisting of more than $50 \%$ fibrous and granulomatous reaction tissues were defined as type B. The fibrous tissues consisted of collagen fiber as shown by Masson trichrome staining.

Patients with types A and B germinomas showed no significant differences in age, sex, location of tumor, or post-biopsy treatment (Table 1). Four patients with type A and one with type B had high pretreatment serum titers of beta-HCG and four patients with type A and one with type B had high pretreatment CSF titers of beta-HCG. No patient had high pretreatment serum and CSF titers of beta-HCG. There were no significant differences in numbers of patients with high serum and CSF titers of beta-HCG between the two groups. All serum and CSF titers of beta-HCG normalized after treatment.

Ten patients with type A germinomas and three patients with type B germinomas were treated with 
Table 2 Radiographic resolution of types $A$ and $B$ germinomas

\begin{tabular}{lcccc}
\hline & \multicolumn{4}{c}{ After treatment ended } \\
\cline { 2 - 5 } & $\begin{array}{c}\text { Within } \\
1 \text { month }\end{array}$ & 3 months & 6 months & 12 months \\
\hline Type A (n = 13) & $13 / 13$ & $13 / 13$ & $13 / 13$ & $13 / 13$ \\
CT (n = 9) & $9 / 9$ & $9 / 9$ & $9 / 9$ & $9 / 9$ \\
MR imaging $(\mathrm{n}=4)$ & $4 / 4$ & $4 / 4$ & $4 / 4$ & $4 / 4$ \\
Type B (n = 5) & $0 / 5$ & $3 / 5$ & $4 / 5$ & $5 / 5$ \\
CT (n = 1) & $0 / 1$ & $1 / 1$ & $1 / 1$ & $1 / 1$ \\
MR imaging $(\mathrm{n}=4)$ & $0 / 4$ & $2 / 4$ & $3 / 4$ & $4 / 4$ \\
\hline
\end{tabular}

CT: computed tomography, MR: magnetic resonance.
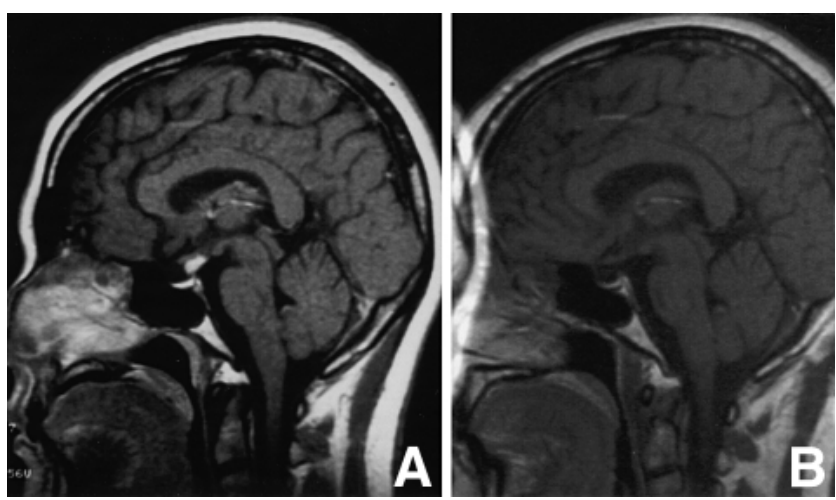

Fig. 3 Pretreatment sagittal $\mathrm{T}_{1}$-weighted magnetic resonance images with gadolinium-diethylenetriaminepenta-acetic acid demonstrating homogeneous enhancement of a type A germinoma in the suprasellar region (A), and disappearance of the mass within 1 month after treatment ended (B).

irradiation only. The other five patients were treated with combined radiotherapy and chemotherapy. There were no significant differences in numbers of patients treated with irradiation only between the two groups.

All type A germinomas (9 studied with CT and 4 with MR imaging) showed radiographic resolution within 1 month after treatment ended (Table 2, Fig. 3). All type B germinomas (1 studied with CT and 4 with MR imaging) persisted at 1 month after treatment ended, but all tumors disappeared within 12 months (Fig. 4).

\section{Discussion}

Radiation exposure induces the death of tumor cells by damaging the deoxyribonucleic acid $^{8)}$ and by disturbing the blood supply. ${ }^{9,17)}$ Since radiosensitive cells are usually dividing rapidly, ${ }^{18)}$ radiation affects

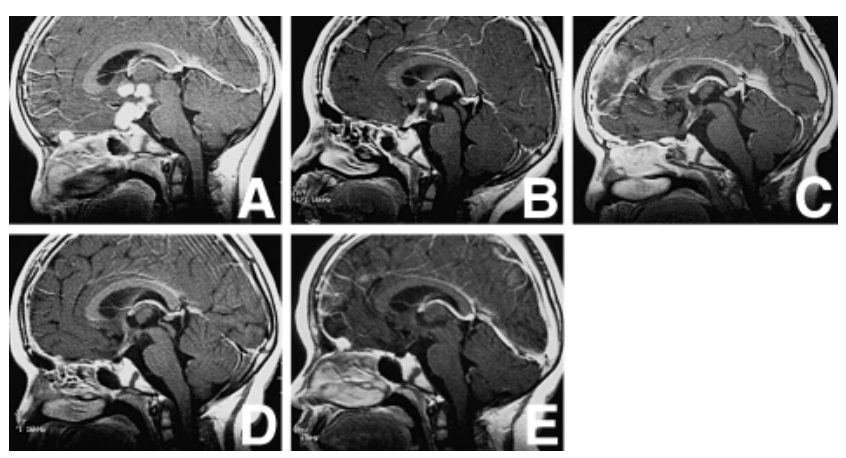

Fig. 4 Pretreatment sagittal $\mathrm{T}_{1}$-weighted magnetic resonance images with gadolinium-diethylenetriaminepenta-acetic acid demonstrating homogeneous enhancement of a type $B$ germinoma in the suprasellar region (A) that persisted for 1 (B), 3 (C), and 6 (D) months after treatment ended, and finally disappeared after 12 months (E).

tumor cells shortly after exposure. In contrast, damage to the vasculature occurs more slowly, and changes may not be appreciated for 6 months to many years after therapeutic radiation. ${ }^{15}$ Other components of tumors show varying sensitivity to radiation: lymphocytes are radiosensitive, ${ }^{13)}$ but interstitial components consisting of reticulin, collagen fiber, and vascular endothelium are not.

Intracranial germinoma consists of large tumor cells with abundant clear cytoplasm, infiltrating lymphocytes, and interstitial components consisting of reticulin, collagen, and vascular endothelium. ${ }^{16)}$ The frequency of granulomatous reaction in intracranial germinoma is $4.7-25 \% .^{1,16)}$ Most specimens of intracranial germinoma contain granulomatous reaction and fibrous tissue, so diagnosis can be difficult. ${ }^{6,10)}$ Such granulomatous reaction and collagen fiber in germinomas is unlikely to disappear in the short term after radiation therapy.

In the present study, type A tumors resolved shortly after radiation, probably due to the relative abundance of radiosensitive oncocytes and infiltrating lymphocyte. In these tumors, little reticulin, collagen, and vascular endothelial component are present. ${ }^{16)}$ In contrast, type B tumors had relatively fewer radiation-sensitive components, oncocytes and lymphocytes, and more radiation-insensitive components, reticulin, collagen, and vascular endothelium. ${ }^{16)}$ This difference may explain why type $\mathrm{B}$ tumors took longer to disappear. ${ }^{11)}$

In large series of intracranial germinoma, $32 \%$ are mixed germ cell tumors. ${ }^{7)}$ The non-germinomatous germ cell tumor components are insensitive to radiation, so could persist after treatment. There- 
fore, if the non-germinomatous components are not appreciated on biopsy, a mistaken histological diagnosis of pure germinoma might be established. Such limited sampling or inadequate review of samples may have led to erroneous claims that some pure germinomas recurred as teratomas ${ }^{12)}$ or immature teratomas. ${ }^{5)}$ Careful follow up with neuroimaging is required to distinguish these persisting nongerminomatous germ cell tumors from type B germinomas, as all type B lesions resolved within 1 year.

The present study indicates that intracranial germinomas with histological evidence of collagen fiber and granulomatous reaction can be expected to resolve more slowly than germinomas lacking these features.

\section{Acknowledgment}

The authors gratefully acknowledge the technical assistance of Miss Yoshie Yasui. We would like to thank Dr. Paul Kretchmer (kretchmer@sfedit.net) at San Francisco Edit (Mill Valley, Calif., U.S.A.) for his assistance in editing this manuscript.

\section{References}

1) Bjornsson J, Scheithauer BW, Okazaki H, Leech RW: Intracranial germ cell tumors: pathobiological and immunohistochemical aspects of 70 cases. J Neuropathol Exp Neurol 44: 32-46, 1985

2) Dattoli MJ, Newall J: Radiation therapy for intracranial germinoma: the case for limited volume treatment. Int J Radiat Oncol Biol Phys 19: 429-433, 1990

3) Friedman JA, Lynch JJ, Buckner JC, Scheithauer BW, Raffel C: Management of malignant pineal germ cell tumors with residual mature teratoma. Neurosurgery 48: 518-523, 2001

4) Jenkin D, Berry M, Chan H, Greenberg M, Hendrick B, Hoffman H, Humphreys R, Sonley M, Weitzman $\mathrm{S}$ : Pineal region germinomas in childhood: Treatment considerations. Int J Radiat Oncol Biol Phys 18: 541-545, 1990

5) Kon H, Kumabe T, Jokura H, Shirane R: Recurrent intracranial germinoma outside the initial radiation field with progressive malignant transformation. Acta Neurochir (Wien) 144: 611-616, 2002

6) Konno S, Oka H, Utsuki S, Kondou K, Tanaka S, Fujii K, Yagishita S: Germinoma with a granulomatous reaction. Problems of differential diagnosis. Clin Neuropathol 21: 248-251, 2002

7) Matsutani M, Sano K, Takakura K, Fujimaki T, Nakamura O, Funata N, Seto T: Primary intracranial germ cell tumors: a clinical analysis of 153 histologically verified cases. J Neurosurg 86: 446-455, 1997

8) McMillan TJ, Tobi S, Mateos S, Lemon C: The use of
DNA double-strand break quantification in radiotherapy. Int J Radiat Oncol Biol Phys 49: 373-377, 2001

9) New P: Radiation injury to the nervous system. Curr Opin Neurol 14: 725-734, 2001

10) Nishibayashi $\mathrm{H}$, Uematsu $Y$, Terada $T$, Itakura $T$ : Pineal germinoma with granulomatous reaction: case report. Neurol Med Chir (Tokyo) 45: 415-417, 2005

11) Ochiai H, Yamakawa $Y$, Fukushima $T$, Sato $Y$, Hayashi $\mathrm{T}$, Yamada $\mathrm{H}$ : Delayed resolution of intracranial germinoma after radiotherapy: a preliminary study of the correlation between histology and magnetic resonance imaging. Neuropathology 20: 190-196, 2000

12) Ono N, Isobe I, Uki J, Kurihara H, Shimizu T, Kohno $\mathrm{K}$ : Recurrence of primary intracranial germinomas after complete response with radiotherapy: recurrence patterns and therapy. Neurosurgery 35: 615-621, 1994

13) Schmitz A, Bayer J, Dechamps N, Thomas G: Intrinsic susceptibility to radiation-induced apoptosis of human lymphocyte subpopulations. Int J Radiat Oncol Biol Phys 57: 769-778, 2003

14) Shibamoto $Y$, Abe $M$, Yamashita J, Takahashi $M$, Hiraoka M, Ono K, Tsutsui K: Treatment results of intracranial germinoma as a function of the irradiated volume. Int J Radiat Oncol Biol Phys 15: 285-290, 1988

15) Tofilon P, Fike J: The radioresponse of the central nervous system: a dynamic process. Radiat Res 153: 357-370, 2000

16) Utsuki S, Oka H, Tanizaki Y, Kondo K, Kawano N, Fujii K: Pathological features of intracranial germinomas with reference to fibrous tissue and granulomatous change. Brain Tumor Pathol 22: 9-13, 2005

17) Valk PE, Dillon WP: Radiation injury of the brain. AJNR Am J Neuroradiol 12: 45-62, 1991

18) von Wangenheim KH, Peterson HP, Schwenke K: Review: a major component of radiation action: interference with intracellular control of differentiation. Int J Radiat Biol 68: 369-388, 1995

Address reprint requests to: S. Utsuki, M.D., Department of Neurosurgery, Kitasato University School of Medicine, 1-15-1 Kitasato, Sagamihara, Kanagawa 228-8555, Japan.

e-mail: utsuki@med.kitasato-u.ac.jp

\section{Commentary}

The authors point out the reasons for a fact that is sometimes observed in clinical practice: why some intracranial tumors, classified histologically as pure germinomas (of the pineal region or not), do not respond to radiation therapy as expected. They con- 
firm what is already known from the literature about the presence of granulomatous reactions in the germinoma tumors, and they demonstrate that if the presence of granulomatous reactions exceeds $50 \%$ of the sample, the germinoma, although pure (in this case type B), will not easily respond to radiation therapy.

It would be interesting to know whether the specimens for the diagnosis had been collected using a stereotactic procedure or an open approach: in the first case, the scarcity of material would increase the difficulty of the diagnosis as usual. Moreover, I would suggest that the authors proceed with their interesting work looking for correspondences between the histological features and the MRI aspects of these tumors, in order to provide pretreatment criteria to evaluate the probability of response.

Alessandro DuCATI, M.D. Ordinario di Neurochirurgia Universita' di Torino Torino, Italy

The authors investigated the histological characteristics of 18 pure germinomas which presented with different responses to radiation therapy. Thirteen tumors disappeared rapidly within 1 month after the completion of radiation therapy, whereas 5 tumors were still observed at the end of radiation therapy and persisted for up to 1 year. In the latter tumors, the authors observed more reticulin or collagen fibers, and vascular endothelium in the tumor tissue as compared to the former tumors. The authors concluded that more collagen fibers or granulomatous reaction in germinoma tissue would be responsible for slow regression of the tumor mass after the radiation therapy.

This paper teaches us a couple of facts useful for the treatment of germinomas. In $20-30 \%$ of germinomas, we could not observe complete tumor disappearance within 1 month after the completion of radiation therapy. Careful histological observation confirming more collagen fibers or granulomatous reaction will be a sign of slow tumor reaction to radiation therapy in germinomas.

Masao Matsutani, M.D., D.M.Sci.
Department of Neurosurgery

Saitama Medical University Saitama, Japan

The authors have performed an important study in which they examined histopathological features that could correlate with patient response to treatment for intracranial germinoma. In the end, they classified germinomas into two types: Type A, the classic twocell pattern germinoma; and type $B$, germinoma with fibrous tissue and granulomatous reaction. The main observation of the study was that the type B germinomas took longer to respond to treatment than did the type A germinomas. These results suggest that germinomas of differing histopathology may have different biological behaviors.

Germinoma remains a highly curable intracranial tumor following most treatment regimens these days with 5 year survivals on the order of $90 \%$. The authors have not provided us with any survival differences between type A and type B tumors, but I assume they were similar. The real value of this study is that the authors have hi-lighted a germinoma subtype that may diminish in size more slowly than the classic germinoma. The treating neurosurgeon and neurooncologist should be aware of this slow response pattern so that concerns about failure to respond to treatment in the short-term can be allayed.

Of course, this is a small series, and a retrospective one. It will be important to duplicate the authors' results in other centers. Furthermore, the segregation of germinoma into these two types will need to be agreed upon by neuropathologists in general. It would indeed be interesting if gene expression profiling could be performed on type A and type B germinomas in the future so that we could understand the molecular basis for the slow response to treatments reported here.

James T. RUtKA, M.D., F.R.C.S.C., F.A.C.S., F.A.A.P. Dan Family Chair in Neurosurgery Division of Neurosurgery The University of Toronto The Hospital for Sick Children Toronto, Ontario, Canada 